\title{
Resenha: Intuition in Judgment and Decision Making
}

\author{
Plessner, H., Betsch, C., \& Betsch, T. (Eds.). (2008). \\ Intuition in judgment and decision making. \\ New York: Lawrence Erlbaum.
}

\author{
Álvaro Machado Dias* \& Avelino Luiz Rodrigues \\ Universidade de São Paulo, São Paulo, Brasil
}

\section{As Bases Epistemológicas da Intuição Decisional}

O ano de 2008 assistiu ao lançamento do primeiro livro de destaque a reunir um corpus de autores especialistas em Tomadas de Decisão de tipo Intuitivo. Trata-se de 'Intuition in Judgment and Decision Making', editado por Henning Plessner, Cornelia e Tilmann Betsch (2008), cujo núcleo contempla as conferências proferidas pelos editores e alguns dos autores convidados, no simpósio de mesmo título, ocorrido na universidade de Heidelberg, Alemanha, em 2004.

O livro possui 325 páginas, dividas em dezenove capítulos independentes, que apresentaremos mais adiante, escritos por acadêmicos de universidades alemãs (i.e. Yver von Cramon, Max Plack Institute; Cornelia Betsch, de Heidelberg) secundados por professores de universidades norte-americanas consagradas (i.e. Seymour Epstein, da Universidade de Massachusetts; Patricia Lindemann, de Columbia).

A publicação coroa esforços recentes para a elucidação dos meandros não declarativos dos processos de tomadas de decisão, reconduzidos à baila, através dos trabalhos de Antonio Damasio e colaboradores sobre a estrutura e função dos marcadores somáticos para decisões irredutíveis ao processamento analítico (Bechara, 2004; Bechara \& Damasio, 2005; Bechara, Damasio, Tranel, \& Damasio, 2005; A. Damasio, 1994; H. Damasio, Bechara, \& Damasio, 2002).

Neste mesmo sentido, 'Intuition in Judgment and Decision Making' imprime mais um passo na constituição de alternativas à tendência hegemônica dos estudos em tomadas de decisão, que é a de se desenvolverem sob a égide do economicismo, em suas duas vertentes fundamentais, a utilitarista e a herística. Apenas a título introdutório, ressalta-se o caráter primordial da Teoria dos Jogos de von Neumann e Morgestern (1944), para a consolidação da vertente utilitarista e as demonstrações

* Endereço para correspondência: Universidade de São Paulo, Faculdade de Medicina, Rua Dr. Ovidio Pires Campos, Cerqueira César, São Paulo, SP, Brasil, CEP 05403-010. Email: alvaromd@usp.br de que em determinadas conjunturas as pessoas não costumam obedecer aos axiomas utilitaristas, para a consolidação da vertente heurística, desde os estudos seminais de Maurice Allais (1955). Para um sumário das contribuições que levaram à queda da hegemonia utilitarista, ver: Allais (1997).

De maneira alternativa a esta maneira de se conceber o processo de tomadas de decisão, esta nova publicação gira em torno da modelagem de sistemas de processamento não-declarativos e não redutíveis a funções discretas. Este conceito de intuição contemplado pelos autores convidados joga luz sobre o efeito de amálgamas afetivo-cognitivos e sensório-cognitivos sobre os processos que ocorrem 'nos bastidores' da definição de escolhas pontuais, contribuindo assim, para alavancar a superação de um verdadeiro tabu científico em relação ao conceito de intuição, o qual passou a servir de limite epistemológico ao ethos racionalista da ciência cognitiva em seus primeiros estágios (intimamente associados ao desenvolvimento teórico dos sistemas físico-simbólicos de processamento de informacional; para uma revisão, ver: Goldstine, 1972).

Não sendo um fato menor, o de que o conceito de intuição verse tanto sobre crenças místico-religiosas, quanto sobre a existência de objetos do conhecimento apenas tangíveis através de processos de conhecimento nãoredutíveis ao encadeamento de representantes de uma sintaxe, o que, diga-se de passagem, está na base da epistemologia ocidental, desde a fundação do platonismo, até a atualidade (para uma revisão, ver: Hart, 1971).

Correlativamente, é de se notar que a superação da relutância em adentrar este campo, reflete a superação de limitações científicas, tais como o restrito conhecimento sobre o processamento de informações no cérebro (i.e. simulação computacional de sistemas de processamento informacional de tipo fuzzy, tipicamente biológicos) e o contraproducente predomínio de concepções inspiradas na metáfora do computador digital e modelos de tomadas de decisões (para modelo clássico, ver: Newell \& Simon, 1972).

Igualmente pertinente ao escopo teórico dos autores deste livro é o desenvolvimento dos estudos do desempe- 
nho decisional de especialistas, vertidos em um conceito estritamente aplicado de intuição, que vicejou ao longo das últimas duas décadas: seja a intuição/expertise de médicos (Leskowitz \& Scott, 2002), enxadristas profissionais (Bilalic, McLeod, \& Gobet, 2007), ou executivos (Ritchie, Kolodinsky, \& Eastwood, 2007), trata-se sempre da capacidade de tornar conhecimentos declarativos acumulados ao longo de anos, em uma estrutura de conhecimento capaz de aumentar o desempenho em novas situações, dotadas de algumas semelhanças com as que se fizeram acumuladas; isto é, de transformar conteúdo em método, na forma de esquemas procedimentais, produzindo com isto uma solução rápida e inédita para problemas urgentes.

Porém, é de se considerar que esta visada não esgota o conceito. Correlativamente, os autores de 'Intuition and Decision Making', vão além da contemplação de ambas as modalidades acima mencionadas e, sobretudo através da colaboração de Epstein (capítulo 2) e dos autores que recorrem à sua obra (capítulos 1, 3, 4, 7 e 16), assumem em parte o desafio de constituir uma metodologia e uma epistemologia para o estudo científico da intuição em tomadas de decisão. Isto é, encaram a proposição e discussão de métodos para a 'elucidação das bases cognitivas da sensação de que uma opção deve ser escolhida, quando não há recursos objetivos para se chegar a tal conclusão'. Não sendo um exagero considerar este como um dos grandes desafios da ciência cognitiva neste século nascente.

\section{Linha Teórica da Obra}

Antes de fazer um apanhado geral dos capítulos, é de se considerar que, no âmbito das teorias atualmente influentes sobre decisões intuitivas, destacam-se três modelos que podem ser definidos como 'plenamente intuitivos': o modelo de processamento Dual de Kahneman e Tversky (Kahneman, 2002, 2003), a Hipótese do Marcador Somático de Damasio e Bechara (Bechara \& Damasio, 2005; Bechara et al., 2005; A. Damasio, 1994) e o modelo CEST (cognitive-experiential self-theory) de Seymour Epstein (Epstein, 1994; Epstein, Pacini, DenesRaj, \& Heier, 1996).

O primeiro, representativo dos estudos denominados Heurísticas e Desvios (Heuristic and Bias), é baseado na perspectiva de que o que se entende por intuição referese a um estágio de pré-processamento decisional, no qual são selecionadas algumas informações essenciais (atenção de cunho não-intencional) derivadas da exposição ao estímulo-chave, em face da qual, uma pré-resposta é resgatada de esquemas de respostas semi-automáticas com tonalidades hedonistas. Em um segundo momento, entraria em ação o processamento analítico, tornando a decisão um ato deliberativo.

De acordo com o Modelo de Damasio e Bechara, o que se entende por intuição decisional se refere ao resgate de memórias sensoriais, relativas às experiências de reforço/punição resultantes de escolhas passadas correlatas, das quais se formaria um sistema de ativação condicionada de dois possíveis tipos, cada um deles composto de duas etapas: 1.a. Similaridade entre um estímulo e outro estímulo (condicionado), associado à punição/recompensa ativaria sistema autonômico e 1.b. Descarga adrenérgica produziria tendência à evitação do estímulo condicionado (circuito 'if'). 2.a. Processo análogo a '1.a.' e 2.b. Similaridade de mesmo cunho ativaria representação mental capaz de transpor a experiência de processamentos somáticos para dentro do sistema nervoso central (circuito 'as if').

Apenas a título introdutório, destacamos que o circuito "if" refere-se às vias que atingem diretamente a somatose (periféricas) e está funcionalmente relacionado à geração condicionamentos do tipo 'ataque e fuga' e o circuito 'as if', fenômeno correlato dentro do cérebro, está funcionalmente relacionado à maximização temporal (para maiores detalhes, ver: Bechara \& Damasio, 2005; Bechara et al., 2005).

De acordo com o modelo de Epstein, a implementação de rotinas decisionais se constituiria do paralelismo irredutível entre a atuação de dois sistemas de processamento de informações: um de tipo declarativo e algorítmico e outro de tipo não declarativo e manifestação afetivo-sensorial (tipo 'experimental'); da interação de ambos emergiriam as tomadas de decisão, através de saídas informacionais deste paralelismo de influências mútuas. Analogamente, quanto maior a tonalidade 'intuitiva' de uma decisão, mais se refletiria a ativação proporcional do sistema experimental e vice e versa (para maiores detalhes ver: Epstein, 1994; Epstein et al., 1996).

O livro em questão é profundamente caracterizado por este último paradigma, que podemos denominar de paradigma da 'intuição holística', o qual evoca a acepção bergsoniana de intuição enquanto 'entendimento que se dá pelos sentidos'. Em termos mais atuais, apesar de representar uma alternativa à hipótese do marcador somático, contrapõem com ainda mais vigor à vertente heurística, cujo ponto de partida é a teoria Prospectiva de Kahneman e Tversky (1979), em relação a qual os editores afirmam: "Neste livro, reunimos trabalhos recentes em Tomadas de Decisão que transcendem a perspectiva atualmente dominante de processos heurísticos" (Plessner, C. Betsch, et al., 2008, p. ix). O sentido maior desta oposição está coadunado à perspectiva de erigir os processos de tipo intuitivo às mais altas instâncias da hierarquia executiva.

\section{Desenvolvimento da Obra}

Dividindo-se em quatro seções: 1. 'A Natureza da Intuição'; 2. 'Aprendizado e Intuição'; 3. 'Emoções e intuição'; 4. 'Vantagens e Desvantagens da Intuição' [tradução nossa], a obra intercala capítulos teóricos e 
experimentais, bem como apresenta ao público mais amplo, a escala PID -Preference for Intuition and Deliberation. Esta escala serve à prospecção de estilos de tomadas de decisão e se correlaciona a medidas de desempenho em situações de pressão (C. Betsch, cap. 14, p. 246-247). Atualmente a mesma está em fase final de validação no Brasil, bem como vem sendo associada a outras escalas de tomadas de decisão, por um dos autores que vos escreve.

A primeira seção contempla os novos modelos teóricos e, por isto, pode ser dita como a mais significativa do livro; formada de cinco capítulos de cerca de dezoito páginas, entre os quais se sobressaem os dois primeiros, escritos respectivamente, pelo editor Tilmann Betsch e por Epstein.

No primeiro, denominado 'A Natureza da Intuição e seu Negligenciamento nas Pesquisas em Julgamento e Tomada de Decisões' [tradução nossa], T. Betsch define a natureza de seu objeto de estudo, ao dizer que todo processo inato de tipo reflexo ou instintivo está fora do escopo da intuição, bem como nem todo tipo de processamento emocional ou pré-cognoscente está incluído, conforme a intuição decisional é um tipo de processamento de escolhas, do qual emerge um sentimento em sentido a uma das opções, o qual não retroage linearmente a etapas de encadeamento lógico-declarativo, mas serve de suporte para a preferência por uma opção em detrimento de outras.

No segundo capítulo, Epstein define seu modelo de sistemas de processamento informacional em paralelo, desenvolvendo o conceito de sistema 'experimental', capaz de dar guarida ao aprendizado automático e a tendências de escolha de tipo rápido, sem esforços e afetivamente direcionadas, em face das quais, emergiria a síntese (dialética) do confronto com o processamento analítico/racional.

A segunda seção, formada por cinco capítulos ligeiramente menores, traz como destaque o capítulo 7. 'A Constituição Automática de Atitudes Implícitas em Relação a Políticos, como Base para o Comportamento Intuitivo do Voto' [tradução nossa]. Neste capítulo, Plessner, T. Betsch, Schallies e Schwieren apresentam os resultados de dois experimentos em escolhas de candidatos fictícios, nos quais se revela o papel preponderante da exposição involuntária à propaganda partidária.

A terceira seção, denominada 'Emoções e Intuição' traz como destaque um capítulo de Haidt e Kesebir, sobre intuições morais ('Na Floresta dos Valores: Porque as Intuições Morais são Diferentes das Outras') [tradução nossa]. Nele, os autores argumentam que "intuições morais são espécies de sistemas sócio-perceptivos que nos permitem ver e sentir a classe dos fatos antropocêntricos" (p. 220); ainda que seja discutível a extensão filogenética do grupo dos animais capazes de comportamentos que pudessem ser denominados morais (i.e. altruísmo entre primatas) e que 'fatos antropocêntricos' seja uma con- cepção que, no limite, favoreça uma definição circular sobre a essência destas intuições/juízos, os autores têm o mérito de restaurar uma acepção importante do conceito, a qual se alinha com as pesquisas recentes em 'bases neurocognitivas das decisões morais', fazendo coro pela justa rejeição do modelo racional de moralidade, em função de modelos francamente afetivos, sejam estes inspirados na existência de módulos específicos para tal categoria de processamento (Garcia \& Ostrosky-Solis, 2006; Moll et al., 2002; Moll, Zahn, de Oliveira-Souza, Krueger, \& Grafman, 2005), ou não (Greene, 2005, 2007).

Na quarta e derradeira seção do livro, 'Os Recursos e os Déficits da Intuição' [tradução nossa], composta por cinco capítulos, destaca-se o capítulo 17, de Unkelbach e Plessner. Neste capítulo, os autores discorrem sobre aspectos e situações que tornam a opção por uma decisão intuitiva menos vantajosa do que a opção por uma decisão analiticamente mediada. A principal conclusão dos autores é a de que uma decisão intuitiva se faz interessante na medida em que a quantidade de informações contemplada pode ser maior do que em uma decisão de cunho analítico - já que esta se restringe pela atenção seletiva - mas, justamente por isto, é provável que informações que não deveriam ser incluídas, permeiem o processo de escolha, prejudicando certas decisões intuitivas.

\section{Considerações Finais}

Sem sombra de dúvidas, este é um livro importante para o campo de estudos em tomadas de decisão, bem como para a ciência cognitiva como um todo, alinhado aos projetos de se desvendar as bases não-declarativas dos processos de escolha, o transfundo quantitativo por trás de saídas informacionais discretas (escolhas), e as maneiras como a afetividade influencia o processamento analítico.

Também aumenta o seu interesse, o fato de ser um livro bem escrito, com capítulos elaborados para trazer ao leitor algum conhecimento objetivo (alguma perspectiva teoricamente essencial e aplicável na prática), de modo que a publicação como um todo possa servir de apanhado geral sobre o assunto.

Em contrapartida, talvez justamente por força desta intenção generalista, o livro sofra de uma ligeira falta de coesão, fragmentada a partir do final da primeira seção. Alternativamente, uma crítica mais incisiva poderia apontar para a relativa fraqueza de certos capítulos, ao menos no que tange à proposta 'informativa' do livro; por exemplo, Plessner, que produziu uma excelente análise de escolhas políticas de cunho intuitivo, contemplando as principais pesquisas no tópico (cap. 7), não se saiu tão bem no capítulo 'Os Benefícios da Intuição' (cap. 15) [tradução nossa], no qual abriu mão de destacar a evidente dicotomia intuição/procrastinação em contexto decisional, vertendo-se sobre conjunturas hipotéticas, que 
parecem servir tão somente ao momento em que se apresentam.

Por fim, é de se notar que ainda falta muito para a integração entre os vários domínios de conhecimento que pontuam este campo (neurociência, modelagem computacional, psicologia experimental, filosofia da mente, etc.), em relação aos quais, a fusão aos modelos de computação biológica de inspiração conexionista (para uma revisão, ver: Mira, 2008) ou simbólico-conexionistas (para uma revisão, ver: Hummel \& Holyoak, 2003) permanece como desafio teórico mais promissor.

Considerando este panorama, nós recomendamos a leitura deste livro, que pode servir de guia tanto à iniciação quanto à atualização dos pesquisadores interessados neste domínio ainda tão pouco delimitado, que é o dos processos não-declarativos em tomadas de decisão.

\section{Referências}

Allais, M. (1955). Fondements d'une théorie positive des choix comportant un risque et critique des postulats et axiomes de l'école américaine: Paris: Imprimerie Nationale.

Allais, M. (1997). An outline of my main contributions to Economic Science. The American Economic Review.

Bechara, A. (2004). The role of emotion in decision-making: Evidence from neurological patients with orbitofrontal damage. Brain and Cognition, 55(1), 30-40.

Bechara, A., \& Damasio, A. R. (2005). The somatic marker hypothesis: A Neural Theory of Economic Decision. Games and Economic Behavior, 52(2), 336-372.

Bechara, A., Damasio, H., Tranel, D., \& Damasio, A. R. (2005). The Iowa Gambling Task and the somatic marker hypothesis: Some questions and answers. Trends in Cognitive Sciences, 9(4), 159-162.

Betsch, C. (2008). Chronic preferences for intuition and deliberation in decision making: Lessons learned about intuition from an individual differences approach. In $\mathrm{H}$. Plessner, C. Betsch, \& T. Betsch (Eds.), Intuition in judgment and decision making (pp. 231-248). New York: Lawrence Erlbaum.

Betsch, T. (2008). The nature of intuition and its neglect in research on judgment and decision making. In H. Plessner, C. Betsch, \& T. Betsch (Eds.), Intuition in judgment and decision making (pp. 3-22). New York: Lawrence Erlbaum.

Bilalic, M., McLeod, P., \& Gobet, F. (2007). Does chess need intelligence? A study with young chess players. Intelligence, 35(5), 457-470.

Damasio, A. (1994). Descartes' Error: Emotion, reason, and the human brain. New York: Avon.

Damasio, H., Bechara, A., \& Damasio, A. R. (2002). Reply to 'do somatic markers mediate decisions on the gambling task?'. Nature Neuroscience, 5(11), 1104-1104.

Epstein, S. (1994). Integration of the cognitive and the psychodynamic unconscious. American Psychologist, 49(8), 709-724.

Epstein, S., Pacini, R., Denes-Raj, V., \& Heier, H. (1996). Individual differences in intuitive-experiential and analytical-rational thinking styles. Journal of Personality and Social Psychology, 71(2), 390-405.
Garcia, A. E. V., \& Ostrosky-Solis, F. (2006). From morality to moral emotions. International Journal of Psychology, 41(5), 348-354.

Goldstine, H. H. (1972). The computer from Pascal to von Neumann. Princeton, NJ: Princeton University Press.

Greene, J. (2005). Emotion and cognition in moral judgment: Evidence from neuroimaging. In J. P. Changeux, A. R. Damasio, W. Singer, \& Y. Christen (Eds.). Neurobiology of human values (pp. 57-66). Berlin, Germany: Springer-Verlag Berlin.

Greene, J. D. (2007). Why are VMPFC patients more utilitarian? A dual-process theory of moral judgment explains. Trends in Cognitive Science, 11(8), 322-323.

Haidt, J. \& Kesebir, S. (2008). In the forest of values: Why moral intuitions are different from other kinds. In H. Plessner, C. Betsch, \& T. Betsch (Eds), Intuition in judgment and decision making (pp. 209-229). New York: Lawrence Erlbaum.

Hart, S. L. (1971). Axiology-Theory of Values. Philosophy and Phenomenological Research, 32(1), 29-41.

Hummel, J. E., \& Holyoak, K. J. (2003). A symbolicconnectionist Theory of Relational Inference and Generalization. Psychological Review, 110(2), 220-264.

Kahneman, D. (2002). Maps Of Bounded Rationality: A perspective on intuitive judgment and choice. Princeton, NJ: Princeton University Press.

Kahneman, D. (2003). Maps of Bounded Rationality: Psychology for Behavioral Economics. The American Economic Review, 93(5), 1449-1475.

Kahneman, D., \& Tversky, A. (1979). Prospect Theory: An analysis of decision under risk. Econometrica, 47(2), 263291.

Leskowitz, E., \& Scott, S. (2002). Medical intuition. In S. Scott (Ed.), Handbook of complementary and alternative therapies in mental health (pp. 269-286). San Diego, CA: Academic Press.

Mira, J. M. (2008). Symbols versus connections: 50 years of artificial intelligence. Neurocomputing, 71(4/6), 671-680.

Moll, J., de Oliveira-Souza, R., Eslinger, P. J., Bramati, I. E., Mourao-Miranda, J., Andreiuolo, P. A., et al. (2002). The neural correlates of moral sensitivity: A functional magnetic resonance imaging investigation of basic and moral emotions. Journal of Neuroscience, 22(7), 2730-2736.

Moll, J., Zahn, R., de Oliveira-Souza, R., Krueger, F., \& Grafman, J. (2005). The neural basis of human moral cognition. Nature Review Neuroscience, 6(10), 799-809.

Newell, A., \& Simon, H. A. (1972). Human problem solving. Englewood Cliffs, NJ: Prentice Hall.

Plessner, H., Betsch, C., \& Betsch, T. (Eds.). (2008). Intuition in judgment and decision making. New York: Lawrence Erlbaum.

Plessner, H., Betsch, T., Schallies, E., \& Schwieren, C. (2008). Automatic online-formation of implicit attitudes towards politicians as a basis for intuitive voting behavior. In $\mathrm{H}$. Plessner, C. Betsch, \& T. Betsch (Eds.), Intuition in judgment and decision making (pp. 107-118). New York: Lawrence Erlbaum.

Plessner, H., \& Czenna, S. (2008). The benefits of intuition. In H. Plessner, C. Betsch, \& T. Betsch (Eds.), Intuition in judgment and decision making (pp. 251-266). New York: Lawrence Erlbaum. 
Ritchie, W. J., Kolodinsky, R. W., \& Eastwood, K. (2007). Does executive intuition matter? An empirical analysis of its relationship with nonprofit organization financial performance. Nonprofit and Voluntary Sector Quarterly, 36(1), 140-155.

Unkelbach, C., \& Plessner, H. (2008). The sampling trap of intuitive judgments: Can reflection reach beyond sampling constraints? In H. Plessner, C. Betsch, \& T. Betsch (Eds.), Intuition in judgment and decision making (pp. 283-294). New York: Lawrence Erlbaum.

von Neumann, J., \& Morgenstern, O. (1944). Theory of games and economic behavior. Princeton, NJ: Princeton University Press. 